\title{
MICROFABRICATED PLANAR PRECONCENTRATOR
}

\author{
Ronald P. Manginell, Gregory C. Frye-Mason, Richard J. Kottenstette, \\ Patrick R. Lewis, and C. Channy Wong \\ Sandia National Laboratories \\ Albuquerque, New Mexico, 87185-1425
}

\begin{abstract}
Front-end sampling or preconcentration is an important analytical technique and will be crucial to the success of many microanalytical detector systems. This paper describes a microfabricated planar preconcentrator ideal for integration with microanalytical systems. The device incorporates a surfactant templated sol gel adsorbent layer deposited on a microhotplate to achieve efficient analyte collection, and rapid, efficient thermal desorption. Concentration factors of 100-500 for dimethyl methyl phosphonate (DMMP) have been achieved with this device, while selectivities to interfering compounds greater than a factor of 25 have been demonstrated. Device performance will be compared with conventional preconcentrators, and the effects of system flow rate, flow channel geometry and collection time will be presented. A physical model of adsorption/desorption from the device will be reviewed and compared with experiment, while numerical simulation of flow over the device will be described.
\end{abstract}

\section{INTRODUCTION}

Chemical preconcentration is a critical analytical procedure since it can purify sample mixtures and boost small analyte concentrations within the range of detectability of a given sensor. This technique is well known in the field of gas chromatography (GC) where a preconcentrator (PC) typically consists of a small tube, often $6 \mathrm{~mm}$ in diameter by $100 \mathrm{~mm}$ long, packed with adsorbent resins between two glass wool plugs. Desired analytes are first collected on the resin by passing a sample mixture through the PC. Then, temperature ramping $\left(\sim 10^{\circ} \mathrm{C} / \mathrm{sec}\right)$ is used to thermally desorb the collected analytes as a plug of gas. The preconcentrator is physically located at the front end of a $\mathrm{GC}$ column and the plug is fed into the $\mathrm{GC}$ for separation into its constituents.

Previously, Kovacs et. al. [1] and Neuhold and Wang et. al. [2] demonstrated electrochemical preconcentration in microfabricated, electrochemical-based detection systems. Their techniques, however, are fundamentally different than that described in this paper. We have developed a microfabricated preconcentrator for gas-phase microanalytical detection systems similar in function to that described above for conventional GC systems. Here, a surfactant templated (ST) sol gel layer, deposited on a microhotplate, is used as the adsorbent and collects analytes from a sample stream directed over its surface. With surface areas as high as $1000 \mathrm{~m}^{2} / \mathrm{g}$ and the ability to tailor surface chemistry and pore size for specific analytes, this class of materials is very attractive as an adsorbent layer. The rapid heating capability of the underlying microhotplate is used to thermally desorb analytes collected in the adsorbent. Heating rates of the order of $10^{4}{ }^{\circ} \mathrm{C} / \mathrm{sec}$ represent at least three orders of magnitude improvement over conventional systems. Given this rapid ramp rate, and, given the low steady-state power consumption $\left(100 \mathrm{~mW}\right.$ at $200^{\circ} \mathrm{C}$, a typical desorption temperature), power consumption in the microfabricated device is at least three orders of magnitude less than conventional systems. These capabilities make this microfabricated design suitable for portable detection systems.

Though useful as a front-end sampling stage for many microanalytical systems, this device has been used primarily in Sandia's $\mu$ ChemLab ${ }^{\top M}$ detection system $[3,4]$. To achieve the goal of rapid (1-3 min), trace detection of target analytes in a hand-held, battery-powered, autonomous system, the $\mu C h e m L a b^{T M}$ relies on three critical microfabricaled components: a deep-etched, siliconbased GC separation column [5], a chemically selective SAW array sensor [6], and the preconcentrator described in this paper.

\section{FABRICATION AND PACKAGING}

The preconcentrator microhotplate has been fabricated by through-wafer silicon etching (via the Bosch process), stopping on a low-stress silicon nitride membrane layer [7]. Prior to silicon etching, a thin-film Pt heater is patterned on the membrane layer on the opposite side of the wafer from the etch window; typically, $1000 \AA$ of Pt and a $150 \AA$ Ti adhesion layer are used (Figure 1 ). Through-wafer etching is preferred to front-side etching for the fabrication of preconcentrator microhotplates, since in the latter embodiment analyte could potentially bypass the adsorptive coating by flowing underneath the membrane. All of the devices described in this paper were Bosch etched. However, wet etchants such as $\mathrm{KOH}$ and TMAH could have been used as well.

The adsorbent ST sol gel layer was applied to the front surface of the microhotplate after the hotplates were etched and the wafer was diced. In most cases, the adsorbent is spray coated, though spin and dip techniques have been demonstrated.

The majority of the test data was taken with a planar preconcentrator mounted in a 24-pin DIP packagc. In this casc, sample flow was directed over the PC by means of a Pyrex lid with a machined flow channel. In the $\mu C h e m L a b^{T M}$ system, an electrical and fluidic circuit board packaging scheme is used [4]. PC performance for both packaging schemes is comparable.

(a)
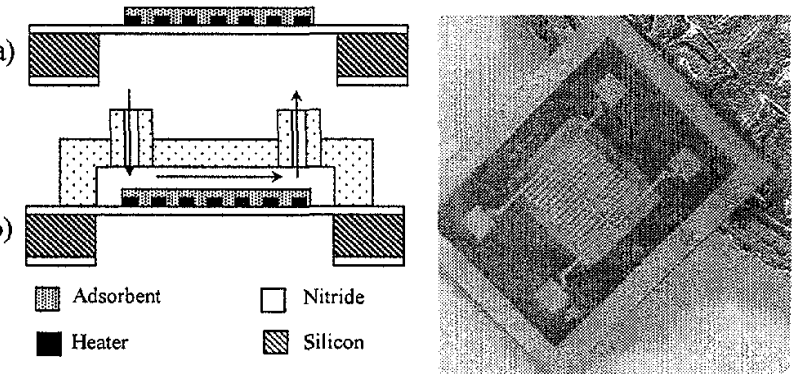

Figure 1. (a) A sectional view of a planar $P C$. (b) The $P C$ with a glass lid used for testing. At right, a completed device on the edge of a quarter. The microhotplate membrane is $2.5 \mathrm{~mm}$ on a side.

\section{DEVICE PERFORMANCE}

The operation of the planar preconcentrator can be broken into two basic steps. First, analytes are selectively collected in the adsorbent by passing a carrier gas (nitrogen) and analyte mixture over its surface. In this step the underlying microhotplate remains in the off, or unheated state. Once sufficient analyte has been collected, the rapid heating capability of the microhotplate is exercised to flash desorb the analyte, returning it to the carrier stream as a purified and concentrated plug. Presented data pertains to DIP packaged PCs with spray-coated adsorbent layers encapsulated by glass flow channels.

Figure 2 demonstrates thermal desorption of dimethyl methyl phosphonate (DMMP) into narrow peak widths, only $200 \mathrm{msec}$ full-width at half maximum (FWHM). The sample was collected by passing $5 \mathrm{ppm}$ of DMMP over the PC for 1 minute. Desorption 
was achieved by pulsing the microhotplate to $200^{\circ} \mathrm{C}$ in $10 \mathrm{msec}$ by the application of a square-wave voltage pulse. Four repetitions of this experiment show reproducibility in peak height and width. Note that the data were renormalized in time to the maximum of one of the curves without loss of generality, since, for a given device, the desorption peak is uniquely determined by the time at which the microhotplate is activated.

The concentration factor $(f)$ specifies the concentration of analyte desorbed relative to the free stream concentration. As might be expected, $f$ is directly proportional to the total collection time $\left(t_{c}\right)$ at a specified flow rate and desorption temperature. For example, for a ST sol gel on a planar PC, collection times of $30 \mathrm{~s}$, $60 \mathrm{~s}$ and $90 \mathrm{~s}$ yield concentration factors of 80,180 and 300 , respectively, for the analyte DMMP. The fact that large values of $f$ can be achieved with short collection times indicates that the planar PC can be used for rapid-response detection systems. Figure 3 illustrates this concept for $50 \mathrm{ppb}$ of DMMP after only $4 \mathrm{sec}$ of collection.

Flow rate also impacts the collection and desorption processes. Generally speaking, collection efficiency is inversely proportional to flow rate. However, desorption peak width is inversely proportional to flow rate due to the relatively larger affect of diffusion processes at low flow. For instance, for a typical $\mathrm{PC}$, a change in flow rate from $6.5 \mathrm{ml} / \mathrm{min}$ to $1.9 \mathrm{ml} / \mathrm{min}$ broadens the peak width from $50 \mathrm{msec}$ to $250 \mathrm{msec}$ FWHM.

The adsorbent coating can be tailored for specific analytes giving high values of $f$ and high selectivity to interferants. Data for the analyte DMMP and two interferants, xylene and methyl ethyl ketone (MEK), are given in Table 1. High selectivity permits lowlevel detection of analytes in real-world environments where interfcrant concentrations can be much greater than that of the analyte (Figure 4), Note that the peak width of Figure 4 was broadened by an in-line, conventional capillary GC column.

Table 1. PC concentration factor and selectivity (relative to $D M M P$ ) for a I minute collection on a $S T$ sol gel coated $P C$.

\begin{tabular}{|c|c|c|}
\hline Compound & $f$ & Selectivity \\
\hline DMMP & 510 & 1 \\
\hline Xylene & 8 & 64 \\
\hline MEK & 18 & 28 \\
\hline
\end{tabular}

The magnitudes of $f$, selectivity, peak width and flow rate described are relevant to the $\mu$ ChemLab ${ }^{T M}$. First, to achieve adequate separation in the $1 \mathrm{~m}$ long microfabricated GC column in a 1-3 minute period with a low-flow miniature pump, the PC must provide pulse widths less than $250 \mathrm{msec}$. The flow generated by the miniature pump for the connection of the PC, GC and SAW detector is in the range of 1 to $3 \mathrm{ml} / \mathrm{min}$. It is important to emphasize that the rapid heating rate of the PC microhotplate is responsible for the narrow peak widths generated, in spite of the low flow through the system. The presented concentration factors are 10-20 times better than those generated with conventional macroscale preconcentrators.

\section{MODELING AND SIMULATION}

Thermal modeling of the preconcentrator microhotplate platform is described in reference [8]. This section summarizes a physical model of adsorption onto, and desorplion from the planar preconcentrator, as well as numerical simulation of flow over the device. The goal of the modeling effort is to direct the PC design for maximal analyte collection (within a specified collection time), while minimizing the temporal width of the desorption peak. The relevant design parameters are the adsorbent area, coating material and the flow channel geometry, particularly its height, to satisfy the performance requirements of the $\mu \mathrm{ChemLab}^{\mathrm{TM}}$.

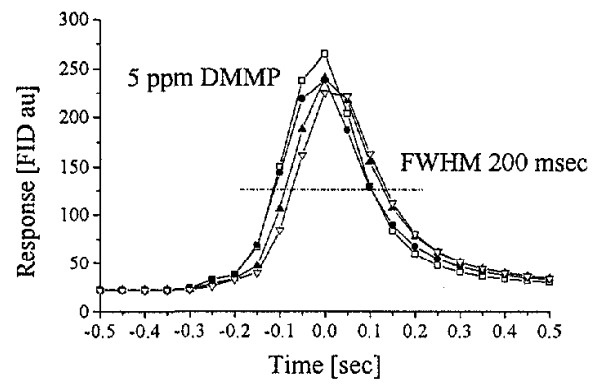

Figure 2. Four repetitions of thermal desorption of DMMP from the planar PC, as detected by a Flame Ionization Detector (FID) in a commercial, benchtop GC system. Flow was $\sim 3 \mathrm{ml} / \mathrm{min}$.

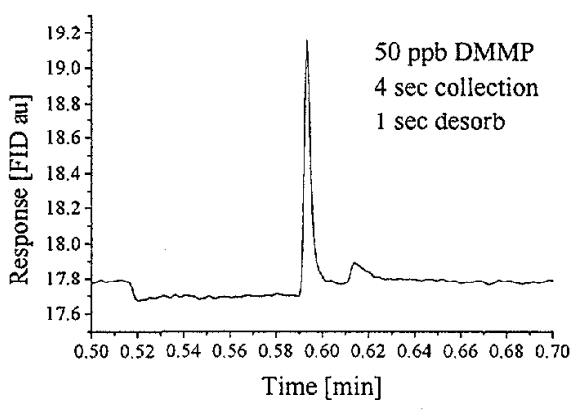

Figure 3. DMMP detection in only $5 \mathrm{sec}$ demonstrating applicability of the $P C$ for rapid response scenarios. The gas flow rate was $\sim 3 \mathrm{ml} / \mathrm{min}$.

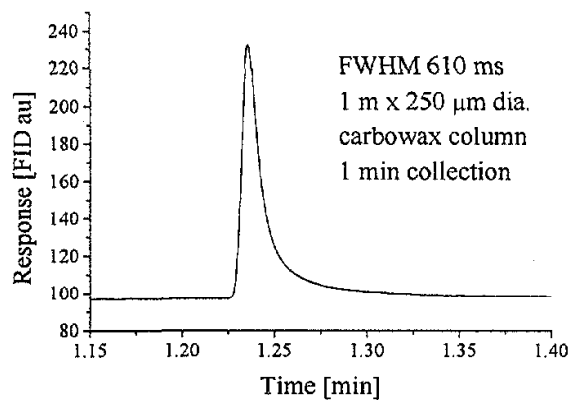

Figure 4. Detection of 50 ppb of DMMP in a 50 ppm background of xylene. Gas flow was $\sim 2 \mathrm{ml} / \mathrm{min}$.

\section{Modeling Adsorption}

The "breakthrough curve" gives the time dependence of the sample concentration eluted from the preconcentrator during adsorption. It must be emphasized that the breakthrough curve is measured downstream from the PC and quantifies the amount of sample that is adsorbed. An analytical model of the breakthrough curve has been developed based on several assumptions: (1) adsorption is irreversible, (2) the adsorption isotherm is linear, (3) the effect of the heat of adsorption is minimal, and (4) the flow in the channel is two-dimensional and laminar, with a parabolic velocity profile. These assumptions simplify the analysis somewhat and lead to an analytical model that illuminates the relative significance of the design parameters. However, the assumptions mandate experimental validation and detailed simulation to support any findings.

The present adsorption model separates the analyte concentration profile in the adsorbent bed into two distinct regions: a saturation zone in which the bed is in equilibrium with 
the feed mixture (analyte and carrier gas) and an adsorption zone in which uptake is still occurring. Prior to the development of a saturation zone at the leading edge of the bed, the breakthrough concentration of the analyte takes on a minimum value given by

$$
c_{\text {min }}=c_{0} \cdot \exp (-\alpha \cdot l), \text { with } \alpha=\frac{k d a}{u h} .
$$

The remaining symbols used are defined in Table 2 . The time required to establish a saturation zone at the front end of the bed is

$$
t_{\text {sat }}=q_{0} \cdot(1-\varepsilon) /\left(\alpha \cdot c_{o} \cdot u\right) \text {. }
$$

As the saturation front propagates across the bed, the breakthrough concentration as a function of load time, $t$, is

$$
c=c_{o} \cdot \exp \left[-\alpha \cdot\left(l-\frac{u \cdot c_{o}}{q_{O} \cdot(1-\varepsilon)} \cdot\left(t-t_{s a t}\right)\right)\right] .
$$

A series of tests have been conducted in which feed concentration, ambient temperature, and channel height have been varied for the purposes of understanding the adsorption and desorption processes, and to evaluate the analytical model. Figure 5 compares the predicted and measured breakthrough curves for adsorption. The model predicts that the breakthrough concentration will rapidly fall to $c_{m i n}$ after adsorption begins at time, $t=0$. The concentration will remain there until the leading edge of the bed becomes saturated. Then, the concentration will increase as the saturation zone propagates across the bed. Evcntually the concentration will return to its initial value when the bed is fully saturated. Observed differences between the predicted and experimental breakthrough curves are mainly attributable to the failure of the model to account for the reversibility of the adsorption process.

\section{Modeling Desorption}

During the thermal desorption process, the release of analyte is assumed to be uniform across the bed, but overall changing with time. (There is no saturation front propagating across the bed.) Analyte is released into the environment due to the change in adsorption equilibrium. When the temperature of the bed is raised, desorption occurs and the concentration of analyte in the adjacent gas phase increases rapidly. The concentration gradient is thus the driving force for diffusion, leading to an increase in concentration of eluted analyte. Desorption will proceed until the concentration of analyte in the gas mixture adjacent to the bed has regained equilibrium with the feed concentration. Hence, it is very important that the model account for the transient behavior of the analyte concentration on the surface of the bed throughout the desorption process.

Taking this transient behavior into account, the breakthrough curve acts as follows: desorption is initiated at $t=0$ when the temperature of the adsorbent is elevated. A sudden sharp spike in the breakthrough concentration is observed, followed by an exponential decay. During desorption the concentration exiting the $\mathrm{PC}$ is given by

$$
c=A(t) \cdot[1-\exp (-\alpha \cdot l)]+c_{o} \exp (-\alpha \cdot l),
$$

where

$$
\begin{aligned}
& p=\frac{k a}{(1-\varepsilon) \cdot\left(q_{0} / c_{1}\right)}, \\
& A(t)=c_{1} \cdot \exp (p t)+c_{0} \cdot[1-\exp (-p t)] .
\end{aligned}
$$

Eqn. (4) shows that the width of the desorption peak is affected by the geometry of the adsorption bed, the adsorption isotherm at the elevated desorption temperature, and the mass transfer coefficient.

The change in the adsorption isotherm with temperature was determined from detailed analysis of desorption data; this analysis is beyond the scope of this paper, but will be provided in a subsequent discourse [9]. Using the derived isotherm, the desorption breakthrough was determined. Figure 6 shows reasonable comparison between the prediction and experiment.

Table 2. Model definitions.

\begin{tabular}{|c|c|}
\hline Symbol & Definition \\
\hline$c$ & analyte concentration exiting PC \\
\hline$c_{o}$ & analyte concentration in the feed stream \\
\hline$k$ & gas-phase mass transfer coefficient \\
\hline$a$ & adsorbent surface area per adsorbent volume \\
\hline$d$ & adsorbcnt laycr thickness \\
\hline$l$ & adsorbent layer length \\
\hline$u$ & average channel flow rate \\
\hline$h$ & flow channel height \\
\hline$c_{l}$ & saturation concentration at desorption temperature \\
\hline$g_{0}$ & analyte concentration in the bed \\
\hline$\varepsilon$ & void fraction \\
\hline
\end{tabular}

\section{Computational Simulation}

Figure 1b illustrates the packaging scheme used here. The width of the channel is $2.8 \mathrm{~mm}$, while the adsorbent is about 2.1 $\mathrm{mm}$ wide. The height of the flow channel is small, in most cases $150 \mu \mathrm{m}$, while for some experiments heights of $50 \mu \mathrm{m}$ and $100 \mu \mathrm{m}$ were used. Finally, gas entry and egress to the lid was accomplished by means of glass capillaries with an inner diameter of $500 \mu \mathrm{m}$. Based on this geometry and usual flow rates, the concern arose that the gaseous flow may be confined to a narrow region between the inlet and outlet. In this scenario, some of the adsorbent area would not be well exposed to the sample stream, leading to degraded collection efficiency. To address this concern, a three-dimensional computational flow simulation using MPSalsa was performed.

MPSalsa is a computer program developed at Sandia for laminar, low Mach number, three-dimensional incompressible and variable density reacting fluid flows; the code is solved on massively parallel computers, using a Petrov-Galerkin finite element formulation. Figure 7 shows the velocity profile in the chamber predicted by MPSalsa. For this geometry, gaseous flow in the chamber is three-dimensional and the flow across the adsorbent is relatively uniform in the transverse direction. This implies that the capture efficiency should be reasonably good and the assumptions of the analytical model are valid.

\section{Modeling and Simulation Summary}

The adsorption and desorption model reviewed here is a useful tool for the optimization of the PC for maximal collection efficiency and minimal desorption peak width. The assumptions required to obtain an analytical solution imply the need for detailed model validation through experiment and simulation. Finally, to numerically model flow over the PC, the MPSalsa code was used. Simulation showed that flow over the adsorbent is relatively uniform for the given geometry and flow conditions.

\section{CONCLUSIONS}

A microfabricated planar preconcentrator has been demonstrated that is ideal for use as a front end in microanalytical and portable systems. The combination of microhotplate technology with high-uptake, tunable adsorbents results in concentration factors and selectivity superior to conventional macroscale devices in many instances, while yielding extremely narrow desorption pulse widths at low power.

An analytical model of adsorption and desorption has been presented that can be used to perform parametric analysis and to optimize the preconcentrator design. The model requires reliable information regarding the characteristics of the adsorbent bed that can be extracted from adsorption/desorption studies. Future correspondence will concentrate on the details of the model and parameter extraction. 


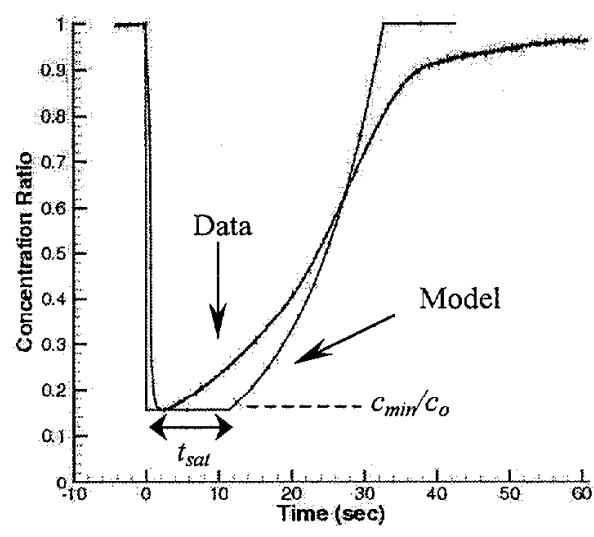

Figure 5. A comparison of the predicted and measured adsorption breakthrough curves. (100 ppm DMMP at $3.3 \mathrm{ml} / \mathrm{min}$.)

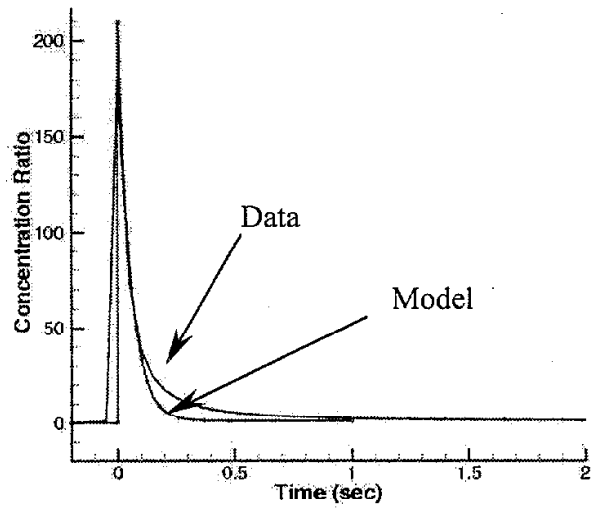

Figure 6. Comparison between the measured and predicted desorption breakthrough curves (100 ppm DMMP at $3.3 \mathrm{ml} / \mathrm{min}$.).

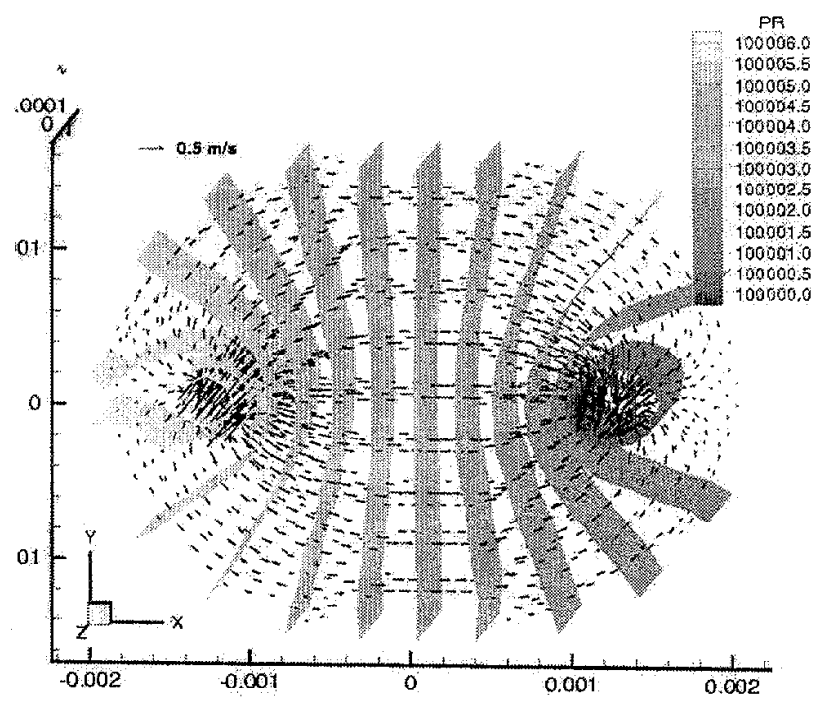

Figure 7. MPSalsa prediction of the distributions of velocity and pressure ("PR" in Pa) in the test flow chamber (150 um height) of the PC. Flow proceeds from the left to the right.

\section{ACKNOWLEDGMENTS}

Sandia is a multiprogram laboratory operated by Sandia Corporation, a Lockheed Martin Company, for the United States Department of Energy under contract DE-AC04-94AL85000. The authors wish to thank C. J. Brinker for the sol gels used herein. All microhotplates were produced at Sandia's Compound Semiconductor Research Lab (CSRL). The fabrication assistance of Sara Sokolowski is gratefully acknowledged.

\section{REFERENCES}

1. G.T.A. Kovaks, C. W. Storment, and S. P. Kounaves, "Microfabricated Heavy-Metal Ion Sensor", Sensors and Actuators $B, 23,41$ (1995).

2. C. G. Neuhold, J. Wang, X. H. Cai, and K. Kalcher, "ScreenPrinted Electrodes for Nitrite Based On Anion-Exhanger-Doped Carbon Inks", Analyst, 120, 2377 (1995).

3. G.C. Frye-Mason, R. J. Kottenstette, E.J. Heller, C. M. Matzke, S.A. Casalnuovo, P.R. Lewis, R. P. Manginell, W. K. Schubert, V. M. Hietala, and R. J. Shul "Integrated Chemical Analysis System for Gas Phasc CW Agent Detcction", Proceedings of the Micro Total Analysis Systems ( $\mu$-TAS) Workshop, Banff, 10/12-16/98, Kluwer, (1998) pp. 477-481.

4. G. Frye-Mason, R. Kottenstette, P. Lewis, E. Heller, R. Manginell, D. Adkins, G. Dulleck, D. Martinez, D. Sasaki, C. Mowry, C. Matzke, and L. Anderson, "Hand-Held Miniature Chemical Analysis System ( $\mu$ Chemlab) for Detection Of Trace Concentrations of Gas Phase Analytes", Proceedings of the $\mu$-TAS '00 Workshop, Enschede, Netherlands, 5/14-18/00. (In press.)

5. C.M. Matzke, S.A. Casalnuovo, G.C. Frye-Mason, R. Kottenstette, R.P. Manginell, D.Y. Sasaki, and C. C. Wong, "Integrated Silicon Gas Chromatographic Micro-Channel", Proceedings of The SPIE Micromachining and Microfabrication Conference, Santa Clara, 9/20-22/98, SPIE Vol. 3511 (1998) pp. 262-268

6. E. Heller, V. Hietala, R. Kottenstette, R. Manginell, C. Matzke, P. Lewis, S. Casalnuovo, G. Frye-Mason, "An Integrated Surface Acoustic Wave-Based Chemical Microsensor Array for Gas-Phase Chemical Analysis Microsystems", Proceedings of the

Electrochemical Society, Honolulu, Hawaii, 10/17-27/99, ECS Vol. 99-23, (1999) pp. 138-142.

7. R. P. Manginell, G. C. Frye, R. J. Shul and C. G. Willison, "Microfabrication of Membrane-Based Devices By HARSE and Combined HARSE/Wet Etching", Proceedings of The SPIE Micromachining and Microfabrication Conference, Santa Clara, 9/20-22/98, SPIE Vol. 3511 (1998) pp. 269-276.

8. R. P. Manginell, D. A. Rosato, D. A. Benson, and G. C. FryeMason, "Finite Element Modeling of a Microhotplate for

Microfluidic Applications", Proceedings of the Second International Conference on Modeling and Simulation of Microsystems, San Juan, Puerto Rico, 4/19-21/99, Computational Publications, Cambridge (1999) pp. 663-666.

9. To be published in the ASME 2000 International Mechanical Engineering Congress \& Exposition, Orlando, FL, 11/5-10/2000. 